\title{
CONSIDERACIONES ACERCA DE LA APLICACIÓN DEL PLAN DE ACCIÓN MUNDIAL SOBRE POBLACIÓN*
}

\author{
VíCTOR L. URQUIDI \\ El Colegio de México
}

El Plan de Acción Mundial sobre Población adoptado en la Conferencia Mundial de Población llevada a cabo en Bucarest en 1974 es un nuevo punto de arranque para considerar los problemas demográficos. Hasta ese momento, los países desarrollados y las organizaciones privadas habían recomendado a los países con alta densidad de población o con altas tasas de incremento demográfico la planificación familiar, incluidos la atención médica, los programas educativos y los de comunicación relacionados con la misma, como elemento casi único de la política de población. Tal política ha sido fuertemente apoyada y presentada como "solución" de peso a los problemas del desarrollo. Este punto de vista fue rechazado en Bucarest y la política demográfica se concibe en la actualidad como un proceso que abarca una extensa variedad de campos de acción, para hacer frente a los efectos y desafíos del cambio demográfico y para identificar las causas de los patrones socialmente inconvenientes de comportamiento demográfico. Por lo tanto, la atención se ha centrado en las condiciones económicas y sociales en las áreas rurales y las urbanas, la migración, el bienestar familiar y la condición de la mujer, así como la salud en general, y en particular la salud materno-infantil.

Además, en el Plan de Acción Mundial sobre Población se subraya el derecho soberano de cada nación a establecer sus propias políticas demográficas y la necesidad de adaptar estas políticas a las circunstancias de cada país. Es evidente que la comunidad internacional no puede imponer políticas a ningún estado miembro en particular: cada país debe analizar sus problemas y desarrollar sus políticas a la luz de sus propios objetivos en los campos económico y social. Es bien sabido que en la elección de políticas intervienen diversas consideraciones, entre ellas el tamaño del país mismo y su relación con los países vecinos, consideraciones políticas, la perspectiva de incrementar la producción de alimentos, la disponibili-

* Una versión preliminar de estos comentarios se preparó para un Grupo Consultivo Interregional de Expertos convocado por el Fondo de las Naciones para Actividades en Materia de Población (UNFPA), con objeto de considerar el Plan de Acción Mundial sobre Población, Ginebra, septiembre 15-19, 1975. Una versión en inglés del actual documento aparece en la revista Population and Development Review. Nueva York, The Population Council, Vol. 2, Núm. 1, marzo de 1976. 
dad de recursos naturales básicos, el grado de industrialización, la naturaleza de las relaciones comerciales y el acceso a los mercados financieros.

Sin embargo, debe aceptarse que la planificación del desarrollo no existe o es rudimentaria en la mayoría de los países en desarrollo y que la percepción de objetivos tal vez no sea muy clara en muchos de ellos. Al ayudar a remediar estas deficiencias, los organismos internacionales pueden hacer una aportación importante para llevar a cabo el Plan de Acción Mundial sobre Población. Los siguientes comentarios procuran esclarecer la naturaleza posible de tal aportación al examinar con brevedad las diferencias regionales existentes y los principales problemas en materia demográfica en los países en desarrollo, y tienen también por objeto identificar prioridades para lograr mejor comprensión de los procesos económico-demográficos, la que es a menudo una precondición decisiva para la adopción de políticas demográficas más racionales.

\section{Multiplicidad del problema}

Para fines demográficos no es útil considerar a los paises en desarrollo en su conjunto. Dejando a un lado ciertos territorios donde se han establecido programas de planificación familiar radicales, debe establecerse una distinción al menos entre las siguientes áreas: $a$ ) Sur y Sudeste asiáticos; $b$ ) Africa al sur del Sahara; $c$ ) el Medio Oriente y Africa del Norte; y d) América Latina. China es un caso especial que no se considerará en este documento.

En el Sur y Sudeste asiáticos, es importante el peso de los números absolutos además de la tasa de crecimiento demográfico prevaleciente, que aún es alta; en esa región el suministro de alimentos es una situación crítica aun a corto plazo. En el caso de Africa al Sur del Sahara, donde son muy altas la mortalidad y la fecundidad, las mágnitudes de la población son relativamente pequeñas (excepto en uno o dos países) y la transición demográfica parece todavía muy distante. En el Medio Oriente, en Africa del Norte y en América Latina, los números absolutos todavía no son críticos pero la tasa elevada de crecimiento demográfico - hasta de 3 y $3.5 \%$ en algunos países- que resulta de la alta fecundidad y una mortalidad decreciente, plantea problemas cuya gravedad puede variar de acuerdo con los recursos básicos y las posibilidades de desarrollo de cada país. En estas áreas, el crecimiento demográfico rápido puede llegar a obstaculizar o aun detener el desarrollo global en la siguiente generación, además de algunas dificultades en los problemas de suministrin de alimentos. Además, en las limitadas posibilidades de empleo influye ya el impacto numérico del crecimiento demográfico veloz de los últimos veinte años sobre el tamaño actual y la tasa de crecimiento de la fuerza de trabajo.

En América Latina en particular, deben tomarse en cuenta sus características culturales especiales, principalmente la influencia de la religión católica en los métodos de planificación familiar disponibles en la actualidad. 
Sin entrar en detalles particulares de las diferentes áreas, baste recordar que las diferencias que acusan requieren distintos enfoques del problema del crecimiento demográfico al tomar en cuenta también las distintas etapas del desarrollo económico, la organización institucional, los factores culturales, etc.

Aun cuando los países del Sur y el Sudeste asiáticos han adquirido experiencia en planificación familiar durante aproximadamente dos decenios. ha variado el éxito de tales programas en forma considerable en las distintas regiones y aun dentro de los mismos países, como es el caso de la India, debido a las diferentes condiciones locales, en particular en relación con la perspectiva del desarrollo económico y social, y frente a factores culturales.

En América Latina se tiene todavía muy poca experiencia en los programas de planificación familiar. En unos cuantos países, en especial en los más pequeños, se han instituido tales programas durante los últimos años, y en uno o dos países de los más grandes se ha empezado el proceso de organizar tales programas. Como se demostró en la Conferencia Regional Latinoamericana de Población de Naciones Unidas efectuada en México en marzo de 1975, todavía se está lejos de llegar a un acuerdo general sobre políticas de población en América Latina. En un extremo están países como Argentina y Uruguay donde, a causa del bajo crecimiento de la población (de acuerdo con los niveles de América Latina, no los europeos) y a causa de la fuerte tradición católica, no existe ningún apoyo a la planificación familiar. Sin embargo, en los últimos años se ha hecho mayor conciencia en otros países de la región en cuanto a que el crecimiento demográfico actual y futuro, junto con la estructura joven de las edades y la migración rural-urbana intensa, traerá consigo graves problemas y desafíos al futuro en términos de empleo, alimentos, educación, salud, desarrollo urbano y oportunidades de vivienda, sobrepoblación rural en las áreas de baja productividad, y demandas para mejorar el bienestar social. Dentro de cada país, solo una fracción relativamente pequeña de la población ha logrado niveles de vida aceptables, y el acceso a todos los servicios sociales (educación, salud, seguridad social, viviendas de bajo costo, etc.), así como el empleo remunerado permanente, se limitan a un pequeño estrato. Es evidente que por el efecto de la fecundidad elevada, aunada al descenso rápido de la mortalidad, es poco probable que las estrategias de desarrollo, implícitas o explícitas, como se han aplicado hasta ahora, conduzcan a una elevación rápida de las condiciones de vida de las mayorías rurales, la clase marginal urbana subempleada o los remunerados de bajos ingresos. Será preciso diseñar nuevas estrategias, lo que de ninguna manera será tarea fácil, muy aparte de los problemas de implementación. Dadas estas condiciones, puede concluirse que la planificación familiar debió haberse introducido hace ya mucho tiempo como elemento de cualquier programa de desarrollo.

Con su abrupto cambio de política en 1973, México ilustra el reciente reconocimiento regional de los problemas demográficos. Las políticas pos- 
tuladas en la nueva Ley de Población, puesta en vigor a principios de 1974 y actualmente en proceso de aplicación, representan un cambio de una posición de indiferencia a una política positiva destinada a reducir la tasa de natalidad. Sin embargo, los voceros del gobierno mexicano tienen mucho cuidado en señalar que la principal tarea es el desarrollo en sí y el asegurar mejores niveles de vida así como oportunidades de empleo. No debe considerarse la planificación familiar como sustituto de los programas de desarrollo o de la ayuda internacional; sólo es parte de una política económica y social racional tomada en su conjunto, que procura ampliar las bases del desarrollo para alcanzar más rápidemente mejores condiciones de vida, en especial para las familias de bajos ingresos. En consecuencia, los programas de planificación familiar que operan a través de los servicios de salud pública, con pleno respeto a los derechos y deseos de los individuos, van acompañados de políticas educativas y de información. Concebidos así en un marco amplio, dichos programas procuran asegurar que a corto plazo tengan rápido acceso a la planificación familiar las mujeres en edad reproductiva en las zonas urbanas y, gradualmente, de acuerdo con la expansión de los servicios de salud, en las áreas rurạles. La cooperación de los organismos internacionales y las organizaciones privadas locales ha tenido buena acogida. Se formó el Consejo Nacional de Población para coordinar y ampliar los diversos elementos del programa. El desarrollo económico y social, en su definición más limitada, corresponde a otras dependencias gubernamentales y no hay interferencia por parte de éstas en los programas de planificación familiar que incluyen esfuerzos coordinados educativos y de comunicación masiva. Sin embargo, se advierte que dentro de este contexto el enfoque del gobierno mexicano es muy cauteloso en el sentido de que las autoridades tienen sumo cuidado de no generar reacciones innecesarias por parte de los grupos conservadores, el clero o los grupos radicales y que, hasta ahora, no ha habido una publicidad abierta en favor de los programas de planificación familiar sino únicamente un llamado a las familias para que consideren muy cuidadosamente su tamaño y su bienestar. Todavía, la información sobre los métodos eficaces de planificación familiar se obtiene en su mayor parte de fuentes no oficiales e informales.

En otros países de América Latina, hay evidencia de tendencias similares o se ha registrado algún progreso, en especial en los países con altas tasas de crecimiento demográfico, tales como los centroamericanos, la República Dominicana, Colombia y Venezuela. Perú y Ecuador no han tomado todavía una acción decisiva en relación con la población, mientras que en Brasil, el país más grande y todavía con una tasa de crecimiento de aproximadamente 3 por ciento anual, se sostiene una posición especial. La existencia en Brasil de un inmenso territorio y ciertas consideraciones políticas han sido factor importante en contra de adoptar oficialmente programas de planificación familiar encaminados a reducir la tasa de crecimiento. Sin embargo, en la conferencia de Bucarest, el representante de Brasil anunció que el gobierno había decidido igualar las 
oportunidades de acceso a la información y los servicios de planificación familiar a todos los sectores de la población, ricos y pobres, aun cuando no se refirió a la tasa de crecimiento como un problema en el desarrollo de Brasil.

El planteamiento de Chile es, de alguna forma, similar al de Brasil, con la diferencia de que por algunos años se ha adquirido alguna experiencia en la implementación de programas de planificación familiar. El caso de Costa Rica es notable, pues la tasa de crecimiento ha descendido considerablemente durante los últimos quince años, con apoyo en los programas oficiales. Barbados, Trinidad y Tabago y Jamaica han adoptado políticas oficiales de descenso de la natalidad; son desde luego islas donde la urgencia de adoptar programas de planificación familiar es mucho mayor, pero todavía son una excepción en las perspectivas de las políticas regionales de América Latina.

Una situación particular, única en América Latina, es la de Cuba, donde se ha hecho caso omiso de consideraciones ideológicas y el gobierno ofrece de hecho todos los métodos de planificación familiar dentro de los servicios de salud, y acepta el aborto voluntario. La tasa de crecimiento demográfico de Cuba no se encuentra entre las más bajas de América Latina pero la fecundidad ha descendido considerablemente desde sus niveles anteriores.

\section{Alcance de la cooperación internacional}

Para ocuparse de los multifacéticos problemas demográficos que se mencionan anteriormente, se requiere una comprensión más profunda de la naturaleza de estos problemas y una capacidad para utilizar tal entendimiento en formular políticas de población más efectivas. Esto requiere de una acción primaria dentro de cada país. Aun cuando no corresponde a los organismos internacionales y las organizaciones privadas interesadas en los problemas de población efectuar los estudios necesarios ni llevar a cabo los programas directamente, pueden sin embargo desempeñar un papel importante. En particular, las organizaciones internacionales pueden estimular la investigación sobre las relaciones entre población y desarrollo y participar en proyectos de desarrollo locales integrados donde las políticas de población entren en juego y puedan constituir un elemento importante. Además de la investigación, las agencias internacionales podrían apoyar diferentes programas experimentales que fueran más allá de la estricta definición de planificación familiar como servicio médico, a fin de permitir a los gobiernos ensayar diversos tipos de programas en los campos educativo y de comunicación. El alcance de tales investigaciones y apoyo programático se comentará a continuación. Algunos de los tópicos mencionados son mandatos del Plan de Acción Mundial sobre Población; otros son extensiones lógicas de propuestas contenidas en ese documento. 


\section{Diseño de políticas}

Un punto de partida obvio al revisar las áreas donde se requiere cooperación es la formulación de políticas nacionales de población. En algunos casos, la cooperación puede requerir apoyo de investigación básica en los diferentes aspectos de población y desarrollo. En otros casos, aun en ausencia de mayor investigación, los países pueden definir como necesaria la reducción de las tasas de fecundidad. El diseño de los programas adecuados puede no obstante ser en extremo difícil debido a la ausencia de conocimientos específicos de las interrelaciones entre las variables socioeconómicas y las demográficas.

En el diseño de las políticas a seguir juegan un papel importante las consideraciones sobre los aspectos institucionales. A este respecto, los países que aún no han adoptado una política de población podrían aprovechar la experiencia de otros países donde las dependencias nacionales pertinentes se han comprometido en la elaboración y ejecución de tales políticas, ya sea en su sentido limitado o como se interpretó en la forma más amplia recomendada en Bucarest.

La comprensión de los problemas de población requiere llegar no sólo a nivel nacional, sino a niveles subnacional, regional y aún local. El éxito que se alcance en el desarrollo de las políticas y los programas de población resultantes puede depender, en algunos países, del grado en que las autoridades, las instituciones académicas y las organizaciones privadas sean capaces de explicar con claridad cuáles son los problemas que trae consigo el crecimiento de la población y su relación con las perspectivas y los planes de desarrollo.

\section{Desarrollo rural}

Debe recordarse que a pesar de una urbanización rápida, la mayoría de los países menos desarrollados son aún predominantemente rurales. De esta forma, las grandes desigualdades de ingreso y de condiciones sociales que prevalecen en las áreas rurales afectan a las grandes mayorías. Parece ser que en general las familias rurales de escasos ingresos no aprecian las posibles ventajas. económicas y sociales de limitar su tamaño. Muy por el contrario, aun sienten que el mayor número de niños es una ventaja en términos de aumentar las posibilidades de obtener ingresos y servicios de trabajo para actividades económicas dentro de la familia rural, ya sea en la agricultura o en el trabajo artesanal. Aun la introducción gradual de la educación refuerza esta actitud, debido a que la familia rural puede considerar que mientras mayor sea el número de niños que ingresen al sistema educativo mayor será la remuneración que con el tiempo perciban para la familia aun emigrando de las áreas rurales. Sea como fuere, la realidad es que las razones de la alta fecundidad o bien las que 
condicionen un posible descenso de la misma en las áreas rurales, no se han explicado lo suficientemente, y poco se sabe acerca de las medidas de política de desarrollo que puedan influir en las actitudes hacia la fecundidad. Fue lamentable que en Bucarest no se haya prestado suficiente atención a este problema. En el Plan de Acción Mundial sólo se hacen breves referencias al desarrollo rural y éstas se dirigen más bien a la necesidad de mejorar las condiciones de vida rurales - "revitalizar el campo"- que al problema particular de las relaciones entre el desarrollo rural y los patrones de fecundidad. ${ }^{1}$

Esto parecería indicar que se debería dar alta prioridad al apoyo a estudios e investigaciones para dilucidar los factores conectados con el desarrollo rural que influyen sobre los patrones de fecundidad y las actitudes respecto al tamaño de la familia. Ello es indispensable para un desarrollo adecuado de los programas de planificación familiar durante los próximos veinticinco años. Tal vez la experiencia de ciertas regiones en la India y otras partes podría ser aprovechada y valorada en forma adecuada por otros países. Sin embargo, deben estudiarse muy cuidadosamente las condiciones locales en cada caso, si se quiere hacer alguna generalización útil. También deben hacerse preguntas específicas sobre la migración rural y su relación con patrones de fecundidad y actitudes alternativos. En algunas áreas, por ejemplo en América Latina, se sostiene ampliamente que una fuerza de trabajo que crezca rápidamente es por sí misma un factor favorables para el desarrollo de la sociedad en su conjunto, pero no se hace suficiente hincapié en las necesidades de capital, los requerimientos de adiestramiento de los recursos humanos o el impacto de la tecnología sobre el empleo. Esto lleva a subestimar el impacto negativo de la alta fecundidad y la fuerte migración rural-urbana sobre las oportunidades futuras de empleo. Es probable que este impacto se amplíe en la medida en que mejore la condición de la mujer y aumente la proporción de mujeres en edades jóvenes y adultas que ingresen a la fuerza de trabajo. En otras palabras, se requiere estudiar el crecimiento de la población, las tendencias del desarrollo rural, la migración y la participación en la fuerza de trabajo como parte de un cuadro integrado.

\section{Fuerza de trabajo y urbanización}

En la mayoría de los países con alto crecimiento demográfico, debe hacerse un estudio mucho más profundo de los aspectos demográficos de la fuerza de trabajo. Esto se aplica en particular a los componentes entre 15 y 29 años de edad, que representan la mayor parte de la fuerza de trabajo que emigra de las áreas rurales a las urbanas. La proporción representada por este grupo de edad está destinada a aumentar o a perma-

1 Véase el Plan de Acción Mundial sobre Población, párrafos 9, $46-49$ (reproducido en Demografía y Economía, Vol. VIII, Núm. 3, 1974, pp. 382-406). También la Recomendación sobre Desarrollo Rural (ibid., pp. 410-412). 
necer elevada durante los próximos 20 a 30 años, y sin duda su número absoluto crecerá de manera considerable en el tiempo. Aunque se han hecho algunos llamados de alerta, pocos países han llevado a cabo estudios profundos acerca del impacto de la migración sobre la fuerza de trabajo urbana y la necesidad de empleos en la estructura urbana. En muchos países de menor desarrollo, las tasas de fecundidad tienden a mantenerse altas en las áreas urbanas a pesar de la disponibilidad de programas de planificación familiar, debido al constante crecimiento de las zonas llamadas "marginales" que están pobladas por migrantes en edad joven que vienen de niveles educativos muy bajos y que habitan en la ciudad con el mismo comportamiento demográfico de la vida rural. Esto se aplica tanto a los hombres como a las mujeres.

Las tendencias hacia la urbanización también requieren de amplios estudios en la mayoría de los países en desarrollo, no sólo en términos de ciudades específicas y sus problemas, sino en términos de toda la estructura nacional de los asentamientos urbanos. Existe necesidad de reorientar las políticas económicas y sociales con relación a la migración interna para estimular el crecimiento de las ciudades pequeñas y medianas, en lugar de las grandes áreas metropolitanas. La experiencia muestra que la mayoría de los gobiernos no adopta políticas de desarrollo urbano a escala nacional sino que delega estos asuntos a las autoridades locales y a las ciudades en particular. Aunque es importante para cada ciudad planificar su desarrollo futuro, también es necesario que determinadas dependencias gubernamentales consideren el problema en su conjunto.

\section{Recursos y medio ambiente}

Las consideraciones a largo plazo sobre las interrelaciones entre población y recursos no recibieron suficiente atención en la Conferencia de Bucarest. Durante los últimos años, se ha efectuado una serie de estudios sobre esta materia, pero todavía a escala global o abarcando grandes regiones conformadas por muy diversos componentes. Los países necesitan ayuda para estudiar sus propias relaciones a largo plazo entre crecimiento demográfico y desarrollo, incluido en especial el impacto del crecimiento de la población sobre el uso y la disponibilidad de los recursos naturales.

\section{Familia y condición de la mujer}

Las consideraciones de política deben dar suficiente atención a las condiciones sociales de la familia, incluidas la condición de la mujer y el impacto de las familias grandes en los niveles de vida familiares, dadas las desigualdades actuales y futuras en el ingreso y la riqueza. La relación entre la distribución del ingreso y los diferenciales de fecundidad apenas ha sido objeto de estudio. Estos temas requieren apoyo para su investi- 
gación y para el diseño de programas de acción que incluyan desarrollo de la comunidad, tanto en el plano urbano como en el rural.

El Plan de Acción Mundial establece el derecho de la pareja a hacer uso de la planificación familiar. Esto incluye aspectos legales, culturales, sociales, económicos y de salud. ¿Qué tipo de programas pueden llevarse a cabo para permitir a las personas ejercer este derecho que en muchos países también ha sido incorporado a la legislación nacional? Los organismos de las Naciones Unidas podrían suministrar útiles servicios a muchos países en sus esfuerzos para determinar las mejores medidas posibles que permitirían, especialmente a los grupos con menores ingresos, hacer uso efectivo de su derecho a la planificación familiar y al espaciamiento de los hijos.

La condición de la mujer en cuanto afecta los factores determinantes del comportamiento de la fecundidad es, también, tema importante de investigacióni y debe considerarse en la formulación de políticas y el diseño de programas. Esto requiere el estudio de factores legales, sociales, culturales y económicos. No hay duda de que esta materia será tomada en consideración por diferentes organizaciones; sin embargo, la relación de la condición de la mujer con los patrones de fecundidad debería ser materia de especial preocupación por parte de los organismos dedicados a población.

\section{Migración internacional}

La migración internacional ha llegado a ser tema de discusión durante los últimos años, debido a las circunstancias particulares que hoy prevalecen, principalmente la migración de los países menos desarrollados a los países altamente industrializados de trabajadores no calificados, por un lado, y de personal técnico y científico, por otro. Debe ayudarse a los países a estudiar el impacto y las causas de la migración de trabajadores y personal técnico y científico, así como a preparar negociaciones para resolver los problemas involucrados mediante acuerdos internacionales. Además de la migración planeada, existe una cantidad considerable de migración ilegal a muchas partes del mundo, pero en especial a Estados Unidos y Canadá. Estos aspectos deben estudiarse con mucho cuidado y deben tomarse medidas para regular dicha migración, a modo que se concilien los intereses de los países de inmigración con los de emigración.

\section{Salud y planificación familiar}

Existe necesidad de mejorar las políticas y los programas de salud en general, sobre todo en el campo de la salud preventiva. Esto incluye, entre otros aspectos, educación para la salud y, desde luego, suministro de cuidados materno-infantiles y servicios de planificación familiar. Esta 
última es necesaria de manera especial en las áreas rurales pero no debe introducirse en forma aislada o en ausencia de servicios de salud. La Organización Mundial de la Salud tiene experiencia considerable en el estudio del problema de la distribución de servicios de salud, incluida la planificación familiar, pero ésta y otras organizaciones podrían obtener mayor experiencia de proyectos experimentales bajo diversas condiciones y deberían activar la difusión de sus experiencias a otros países.

A pesar de la enorme cantidad de investigaciones llevadas a cabo sobre reproducción humana y técnicas contraceptivas durante los últimos quince años, subsiste la necesidad de investigaciones adicionales en este campo. Debido a que las dos formas principales de anticonceptivos - la píldora y los dispositivos intrauterinos- no son del todo satisfactorios, se requiere desarrollar métodos adicionales que sean más efectivos, más fáciles de aplicar y mejor adaptados a las condiciones culturales, en especial en las áreas rurales. Los acuerdos de cooperación entre institutos de investigación de los países desarrollados y los países en vía de desarrollo podrían ser más útiles aumentando la transferencia de investigación a estos últimos, junto con el adiestramiento del personal de investigación. Debe animarse a los países de menor desarrollo a producir anticonceptivos tanto para su venta a precios bajos por la vía comercial como para su distribución gratuita a través del sistema de salud pública.

El Plan de Acción Mundial sobre Población evadió el problema dẹl aborto. Es bien sabido que en los países de menor desarrollo, se practica el aborto en forma clandestina bajo condiciones peligrosas para la salud y aun la vida de las mujeres. Debe apoyarse a los países para que efectúen estudios sobre la magnitud del aborto ilegal, las circunstancias en que se practica, sus características y la relación de la planificación familiar con la reducción de los abortos inducidos. Sin embargo, debe estimularse también a los países a considerar modificaciones en su legislación para liberalizar el aborto no como método principal de planificación familiar, sino como remedio en los casos en que las medidas contraceptivas no hayan sido efectivas, y como medio de evitar los peligrosos abortos inducidos clandestinos.

Dentro del campo de la salud, el mayor énfasis de las políticas de población se debe colocar, desde luego, en los programas mismos de planificación familiar. Deberían asignarse mayores recursos a los países de menor desarrollo para diseñar los programas, organizarlos en forma adecuada, efectuar el adiestramiento del personal médico y paramédico necesario, llevar a cabo programas educativos relacionados con la planificación familiar y, en especial, ofrecer estos servicios en las áreas rurales.

\section{Recolección de datos, investigación, adiestramiento y evaluación}

El Plan de Acción Mundial sobre Población ofrece importantes lineamientos en materia de recolección y análisis de los datos. Es necesario 
hacer notar la necesidad del apoyo continuo a este tipo de trabajos, en particular en los países de menor desarrollo. El Plan señala también la necesidad de efectuar investigación y adiestramiento. En general, no es aconsejable que agencias privadas extranjeras efectúen investigaciones sobre políticas de población, sino que debieran ocuparse de ellas directamente las autoridades locales y las instituciones académicas del país. Así resulta especialmente importante reforzar la capacidad de investigación en los países de menor desarrollo. Debe desecharse cualquier intento inadecuado de aplicar los resultados de investigación relativos a una área dada a condiciones locales distintas. También en las investigaciones debe hacerse mayor énfasis en los métodos interdisciplinarios. Debe ampliarse el alcance y la duración de las becas de adiestramiento de posgrado en el exterior y aun dentro de los países de menor desarrollo. Además del apoyo a los institutos nacionales o locales, deberían reforzarse los centros regionales de Naciones Unidas para investigación y adiestramiento en materia de población (CELADE, etc.).

Se precisa de un apoyo continuo a los programas de adiestramiento para personal médico, paramédico y de otra índole que se requiere para habilitar tales políticas, así como a la preparación de material educativo y de adiestramiento en la administración de proyectos y programas de planificación familiar y salud. Todavía se ha dado muy poca atención al adiestramiento de administradores en cuanto a las grandes implicaciones sociales, demográficas y económicas del crecimiento de la población. Esto puede remediarse con el apoyo de cursos adecuados de formación, seminarios, etc. Algunas de estas actividades no deben restringirse a programas de nivel nacional, sino que pueden organizarse a nivel regional o subregional.

A medida que los programas de planificación familiar logren algún avance y que se habiliten las políticas de población dentro de un contexto más amplio en los países menos desarrollados, existirá la necesidad de vigilar los programas y evaluarlos continuamente. A su vez, esto requiere de mejores métodos y de un adiestramiento específico adecuado. La evaluación no debiera ser sólo numérica, en términos de ciertos objetivos deseados o tasas de costo-beneficio, sino también cualitativa, que incluya el examen de todo el espectro de las repercusiones de las políticas y los programas de población en el desarrollo.

\section{La necesidad de conocimientos y la acción: dilema de sincronización}

Las consideraciones anteriores muestran la complejidad de los problemas de población, en particular como se observan a la luz de la Conferencia de Bucarest, y aluden a la gran variedad de campos en los que la cooperación internacional podría contribuir a su solución. Algunas veces resulta peligroso embarcarse en políticas sin la suficiente investigación o comprensión de los problemas relacionados con tal política; dilema que 
afronta la mayoría de los países. Sin embargo, aun cuando no exista una comprensión global de todos los problemas, en muchos países de menor desarrollo puede percibirse con claridad que, dadas las tendencias a largo plazo, las elevadas tasas de crecimiento demográfico tienen en efecto graves repercusiones para el futuro. Este es el caso particular en tanto no se disponga de grandes avances técnicos, en especial en la producción de alimentos, y mientras no se efectúe algún cambio importante en las actitudes de las familias hacia el espaciamiento y el número de los hijos, sobre todo en las áreas rurales. La investigación, aun aquella basada en encuestas directas, toma tiempo para planearse, efectuarse y aun evaluarse. Para muchos países tal vez sea demasiado tarde para llevar a cabo complejas investigaciones previas sobre su crecimiento demográfico, cuando el período de duplicación de la población se efectúa en lapsos tan reducidos como de 20 y 25 años, y cuando ya experimentan el fuerte impacto del crecimiento demográfico rápido sobre el empleo de la fuerza de trabajo actual. A pesar de la retórica empleada en el Plan Mundial de Acción sobre Población, no será posible para muchos países sostener por mucho tiempo una elevada tasa de crecimiento demográfico, cualesquiera que sean los cambios económicos y sociales considerados como necesarios para redondear políticas de población y de desarrollo a largo plazo bien concebidas. Por consiguiente, se justifica que algunos países inicien y favorezcan a la brevedad posible una reducción de la fecundidad a través de los medios disponibles. En estas condiciones, es importante que la experiencia internacional esté a disposición de cualquier país que tome decisiones en pro de reducir la fecundidad, a fin de que las deficiencias de los programas anteriores se eviten en la medida de lo posible. 